\title{
Editorials
}

\section{Fat embolism and postoperative coagulopathy}

\author{
Robert J. Byrick MD
}

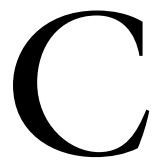

ARDIOPULMONARY instability during cemented hip arthroplasty continues to occur. The incidence of intra- operative cardiac arrest, often attributed to pulmonary fat microembolism, is estimated at $0.6 \% .^{1,2}$ Transesophageal echocardiography demonstrates echogenic material in the right ventricle in over $90 \%$ of patients having total hip arthroplasty, yet few patients have any clinical signs or symptoms of fat embolism. ${ }^{3}$ The clinical relevance of these embolic events remains controversial. What factors determine whether a patient experiences clinically significant consequences from the 'intravasation' of marrow products during orthopedic surgery?

In 1997, the Anesthesia Advisory Committee to the Chief Coroner of Ontario reported two intraoperative deaths during hip replacement for fractured femur. ${ }^{4}$ After reviewing these cases, the Committee emphasized two clinically important points. Firstly, patients with limited cardiopulmonary reserve appear at highest risk, thus anesthesiologists should anticipate the possibility of sudden cardiac arrest. Secondly, they strongly recommended that aortic perfusion pressure be maintained, using vasoconstrictors if necessary, to preserve right ventricular perfusion and function. Experimental models clearly showed that systemic hypotension is caused by acute pulmonary hypertension with secondary right ventricular dilation and failure. ${ }^{5}$ The hypotension with decreased cardiac output results from a right to left interventricular septal shift and consequently, decreased left ventricular end-diastolic volume. In the report by Fallon et al. in this issue of the journal, such a resuscitation protocol was generally followed with restoration of hemodynamic function. ${ }^{6}$ After resuscitation, however, diffuse pulmonary edema, coagulopathy and refractory shock resulted in death. The authors report that "autopsy confirmed the cause of death as fat embolism" ${ }^{6}$ Was intravascular fat really the cause of death or does the postmortem presence of fat represent an epiphenomenon: a consistent occurrence, which does not have a causative relationship to the disease process?

The "intravasation" of fat and marrow during orthopedic surgical procedures requires three factors. There must be a disruption of vessels within the medullary cavity, particulate fat and marrow in the cavity after reaming, and there must be pressurization of the canal. In an animal model, we showed that lavage of the medullary canal prior to pressurization prevents cardiopulmonary instability and reduces the amount of intravascular fat in the lung. ${ }^{7}$ This has been verified in clinical studies that modified the surgical technique. ${ }^{3}$ The primary role of methylmethacrylate cement appears to be as a sealant of the medullary cavity, which results in high medullary canal pressures during insertion of the femoral component, forcing marrow contents into the venous system. Autopsy findings often confirm the presence of intravascular fat, thrombin and cellular marrow products in the lungs of patients who die immediately after cemented arthroplasty. Echocardiography cannot distinguish between fat, marrow debris, air or other particulate material.

Modig studied the relationship between fat embolism and the coagulation system and stated that "the presence of fat particles in the pulmonary circulation could be regarded as an indicator of release, not only of bone marrow fat, but also of other tissue thromboplastins". ${ }^{8}$ Dahl et al. showed that increased generation of thrombin- antithrombin III complexes precede the use of bone cement during hip surgery, suggesting that reaming is associated with intravasation of marrow products (including fat), which can activate the coagulation cascade. 9 Barie and Malik used a bone marrow infusion model to study the role

From the Department of Anaesthesia, St. Michael's Hospital, University of Toronto, Toronto, Ontario, Canada.

Address correspondence to: Dr. Robert J. Byrick, Department of Anaesthesia, St. Michael's Hospital, 30 Bond Street, Toronto, Ontario M5B 1W8, Canada. Phone: 416-864-5071; Fax: 416-864-6014; E-mail: robert.byrick@utoronto.ca. 
of intravascular coagulation and granulocytes in acute lung injury. ${ }^{10}$ They showed that bone marrow contains thromboplastin, which activates the extrinsic coagulation system, resulting in decreased plasma fibrinogen concentration and increased fibrin degradation products. Interestingly, they also suggested that the amount of fat in marrow could not account for the large increase in pulmonary artery pressure and that secondary microvascular thrombosis was the cause. Importantly, they showed that bone marrow infusion did not produce pulmonary hypertension in defibrinogenated sheep. In their experiment, activation of intravascular coagulation was required for the increase in lung vascular permeability and pulmonary edema. ${ }^{10}$

Most patients are easily resuscitated after intraoperative hypotension during cemented arthroplasty. Most recover uneventfully, as the acute hemodynamic changes are "time-limited". Even after cardiac arrest and resuscitation, such an impressive coagulopathy, leading to shock and death is exceedingly rare. ${ }^{6,11}$ Why should such an atypical and extreme response to a cemented joint replacement occur in this patient? 6 Persistent low cardiac output, with tissue hypoperfusion could cause a secondary coagulopathy, leading to refractory shock and death. Another possible explanation is related to the fact that this hip arthroplasty was done to treat a pathologic hip fracture (not an elective arthroplasty for osteoarthritis). ${ }^{6}$ Whether pathologic fractures have abnormal vessels that predispose to the 'intravasation' of marrow products has not been established. It is possible that tumour fragments or abnormal tumour products were forced into the vascular system at the time of prosthesis insertion, to stimulate a massive activation of coagulation. If the pathophysiology described by Barie and Malik is correct, activation of coagulation with thrombin deposition could account for the prominence of pulmonary hypertension and subsequent lung edema, followed by disseminated intravascular coagulopathy in this patient. Adipose tissue is said to contain thromboplastin. ${ }^{2}$ Whether intravascular fat is sufficient, or even necessary, to cause this coagulopathy remains unknown. This is an important point, since over $90 \%$ of patients have detectable echogenic emboli during routine cemented arthroplasty and autopsy summaries continue to assume a cause-effect relationship between the intravascular fat and the pulmonary and systemic consequences of cemented arthroplasty. This case serves to remind us that intravascular fat may be only a marker for the 'intravasation' of marrow products during cemented arthroplasty. More research is needed before a direct causative link can be confirmed.

\section{L'embolie graisseuse et la coagulopathie postopératoire}

L'instabilité cardio-pulmonaire survient encore pendant l'arthroplastie de la hanche cimentée. L'incidence d'arrêt cardiaque peropératoire, souvent attribué à une micro-emoblie graisseuse pulmonaire, est estimée à 0,6 \%. ${ }^{1,2}$ L'échocardiographie transœesophagienne démontre la présence de matériel échogène dans le ventricule droit de plus de $90 \%$ des patients qui subissent une arthroplastie totale de la hanche, néanmoins peu de patients présentent des signes ou des symptômes cliniques d'embolie graisseuse. ${ }^{3}$ La signification clinique de ces incidents emboliques demeure controversée. En effet, quels facteurs déterminent si un patient va subir les conséquences, significatives au plan clinique, de "l'intravasation" de produits de la moelle survenue pendant une intervention orthopédique?

En 1997, le Comité consultatif auprès du coroner en chef de l'Ontario a signalé deux décès survenus pendant l'arthroplastie de la hanche consécutive à une fracture du fémur. ${ }^{4}$ Après révision, le Comité a souligné deux aspects cliniques importants. Premièrement, les patients qui présentent une réserve cardio-pulmonaire diminuée semblent plus à risque, ce qui devrait alors inciter les anesthésiologistes à prévoir la possibilité d'un arrêt cardiaque soudain. Deuxièmement, on recommande que la pression de perfusion aortique soit maintenue, en utilisant des vasoconstricteurs si nécessaire, pour préserver la perfusion et la fonction du ventricule droit. Les modèles expérimentaux ont clairement montré que l'hypotension généralisée est causée par une hypertension pulmonaire aiguë et par une dilatation et une insuffisance secondaires $\mathrm{du}$ ventricule droit. ${ }^{5}$ L'hypotension accompagnée d'une baisse du débit cardiaque est le résultat d'un déplacement septal de la droite vers la gauche et, en conséquence, d'une diminution du volume télodiastolique du ventricule gauche. Dans l'article de Fallon et coll., le protocole de réanimation mentionné est habituellement suivi d'une restauration de la fonction hémodynamique. ${ }^{6}$ Cette fois, après la réanimation, un œè̀me pulmonaire diffus, une coagulopathie et un choc réfractaire ont provoqué le décès du patient. Les auteurs indiquent que "l'autopsie a confirmé que l'embolie graisseuse avait causé le décès". 6 La graisse intravasculaire est-elle vraiment la cause de la mort ou la présence de graisse cadavérique est-elle un 
épiphénomène : une occurrence constante qui n'a pas de relation causale avec le processus morbide?

"L'intravasation" de graisse et de moelle pendant l'intervention orthopédique dépend de trois facteurs. On doit retrouver une rupture des vaisseaux à l'intérieur de la cavité médullaire, des particules de graisse et de moelle dans la cavité après l'alésage de l'os et une pressurisation du canal. Dans un modèle animal, nous avons montré que le lavage du canal médullaire réalisé avant la pressurisation prévient l'instabilité cardio-pulmonaire et réduit la quantité de graisse intravasculaire dans le poumon. ${ }^{7}$ Cela a été vérifié dans des études cliniques qui ont modifié la technique chirurgicale. ${ }^{3}$ Le ciment de méthyle méthacrylate agit principalement comme un produit d'étanchéité de la cavité médullaire, ce qui entraîne de fortes pressions dans le canal médullaire pendant l'insertion de la composante fémorale, poussant le contenu médullaire vers le système veineux. Les résultats d'autopsie confirment souvent la présence de graisse intravasculaire, de thrombine et de cellules médullaires dans les poumons des patients qui décèdent immédiatement après l'arthroplastie cimentée. L'échocardiographie ne permet pas de distinguer entre la graisse, les débris médullaires, l'air ou d'autres particules de matières.

Modig a étudié la relation entre l'embolie graisseuse et le système de coagulation et il a indiqué que "la présence de particules graisseuses dans la circulation pulmonaire pourrait être un indicateur de la libération, non seulement de la graisse médullaire, mais aussi d'autres thromboplastines tissulaires". ${ }^{8}$ Dahl et coll. ont montré que la reproduction accrue de complexes thrombines-antithrombines III survient avant l'utilisation de ciment osseux pendant l'opération de la hanche, ce qui laisse croire que l'alésage est associé à la pénétration intravasculaire de produits médullaires, y compris des graisses, pouvant activer la cascade de la coagulation. Barie et Malik ont utilisé un modèle d'injection de moelle osseuse pour étudier le rôle de la coagulation et des granulocytes intravasculaires dans le cas de lésion pulmonaire aiguë. ${ }^{10} \mathrm{Ils}$ ont montré que la moelle osseuse contient de la thromboplastine qui active la coagulation exogène, ce qui entraîne une baisse de la concentration plasmatique de fibrinogène et une augmentation des produits de dégradation de la fibrine. Autre fait intéressant, la quantité de graisse dans la moelle ne pourrait expliquer l'importante augmentation de pression artérielle pulmonaire et la thrombose microvasculaire secondaire serait la cause. Ils ont montré, et c'est important, que l'injection de moelle osseuse n'a pas produit d'hypertension pulmonaire chez des moutons défibrinés. Leur expérience comportait l'activation de la coagulation intravasculaire pour produire l'augmentation de la perméabilité vasculaire et de l'œdème pulmonaires. ${ }^{10}$

La plupart des patients sont facilement réanimés à la suite d'hypotension survenue pendant l'arthroplastie cimentée. La récupération se déroule bien en général, puisque les changements hémodynamiques aigus sont "limités dans le temps". Même après un arrêt cardiaque et une réanimation, une coagulation d'une telle importance menant au choc et à la mort est extrêmement rare. ${ }^{6,11}$ Pourquoi une réaction aussi atypique et extrême au remplacement d'une articulation s'est-elle produite chez ce patient ${ }^{6}$ La persistance d'un faible débit cardiaque, accompagné d'hypoperfusion tissulaire, pourrait causer une coagulopathie secondaire et provoquer un choc réfractaire et la mort. Une autre explication possible est reliée au fait qu'il s'agissait d'une arthroplastie de la hanche réalisée pour traiter une fracture de hanche pathologique, non une arthroplastie planifiée pour ostéoarthrose. ${ }^{6}$ Quant à la possibilité que les fractures pathologiques présentent des vaisseaux anormaux qui prédisposeraient à "l'intravasation" de moelle, cela n'a pas encore été prouvé. Il est possible que des fragments de tumeur ou du tissu tumorale anormal aient été poussés dans le système vasculaire au moment de l'insertion de la prothèse et qu'ils aient stimulé une activation massive de la coagulation. Si la physiopathologie décrite par Barie et Malik est exacte, l'activation de la coagulation avec dépôt de thrombine pourrait expliquer l'importance de l'hypertension pulmonaire, et l'œdème pulmonaire subséquent, suivi d'une coagulopathie intravasculaire disséminée chez ce patient. On dit que le tissu adipeux contient de la thromboplastine. ${ }^{12}$ Mais on ne sait toujours pas si la graisse intravasculaire est suffisante, ou même nécessaire, pour causer cette coagulopathie. C'est un point important, puisque plus de $90 \%$ des patients ont une embolie échogène détectable pendant l'arthroplastie cimentée de routine et les rapports d'autopsie supposent encore une relation de cause à effet entre les graisses intravasculaires et les conséquences pulmonaires et généralisées de l'arthroplastie cimentée. Ce cas nous rappelle que les graisses intravasculaires pourraient n'être qu'un marqueur de "l'intravasation" de produits de la moelle pendant l'arthroplastie cimentée. D'autres recherches sont nécessaires pour confirmer un lien de cause à effet.

\section{References}

1 Dabl OE, Molnar I, Rø JS, Vinje A Global tests on coagulation and fibrinolysis in systemic and pulmonary circulation accompanying hip arthroplasty with acrylic cement. Thromb Res 1988; 50: 865-73. 
2 Woo R, Minster GJ, Fitzgerald RH, Mason LD, Lucas $D R$, Smith FE. The Frank Stinchfield award.

Pulmonary fat embolism in revision hip arthroplasty. Clin Orthop 1995; 319: 41-53.

3 Koessler MJ, Fabiani R, Hamer H, Pitto RP. The clinical relevance of embolic events detected by transesophageal echocardiography during cemented total hip arthroplasty: a randomized clinical trial. Anesth Analg 2001; 92: 49-55.

4 Pietak S, Holmes J, Matthews R, Petrasek A, Porter B. Cardiovascular collapse after femoral prosthesis surgery for acute hip fracture. Can J Anaesth 1997; 44: 198-201.

5 Murphy P, Edelist G, Byrick RJ, Kay JC, Mullen JB. Relationship of fat embolism to haemodynamic and echocardiographic changes during cemented arthroplasty. Can J Anaesth 1997; 44: 1293-1300.

6 Fallon KM, Fuller JG, Morley-Forster P. Fat embolization and fatal cardiac arrest during hip arthroplasty with methylmethacrylate. Can J Anesth 2001; 48: 626-29.

7 Byrick RJ, Bell RS, Kay JC, Waddell JP, Mullen JB. High-volume, high-pressure pulsatile lavage during cemented arthroplasty. J Bone Joint Surg 1989; 71: 1331-6.

8 Modig J, Busch C, Olerud S, Saldeen T. Pulmonary microembolism during intramedullary orthopaedic trauma. Acta Anaesthesiol Scand 1974; 18: 133-43.

9 Dahl OE, Johnsen H, Kierulf P, et al. Intrapulmonary thrombin generation and its relation to monomethylmethacrylate plasma levels during hip arthroplasty. Acta Anaesthesiol Scand 1992; 36: 331-5.

10 Barie PS, Malik AB. Role of intravascular coagulation and granulocytes in lung vascular injury after bone marrow embolism. Circ Res 1982; 50: 830-8.

11 Byrick RJ, Forbes D, Waddell JP. A monitored cardiovascular collapse during cemented total knee replacement. Anesthesiology 1986; 65: 213-6.

12 Saldeen $T$. Trends in microvascular research. The microembolism syndrome. Microvasc Res 1976; 11: 227-59. 